\title{
Intelligent Solar Tracker Vehicle for Supply Channel of Electric Vehicle Need Using Image Processing and Mobile Network
}

\author{
Smitha.S.M ${ }^{1}$, Goutham S Tantri ${ }^{2}$, Manjunatha.M. J ${ }^{2}$, Vardhan.Ch. ${ }^{2}$, Gagan.G. L ${ }^{2}$, Vijaykumar G Naik ${ }^{2}$. \\ ${ }^{1}$ Assistant Professor: Department of ECE, Jawaharlal Nehru National College of Engineering. Shivamogga, India. \\ ${ }^{2}$ UG Student: Department of ECE, Jawaharlal Nehru National College of Engineering, Shivamogga, India.
}

\begin{abstract}
Harnessing the renewable energy sources in efficient way is the present day need when the nonrenewable sources are depleting in alarming rate. Many available renewable sources are hardly harnessed due to high conversion cost and sophisticated technical need. Solar is one of the reliable renewable resource and it is environment friendly and easy to convert into other forms i.e. heat and electric. The electricity generation in solar is based on silicon technology and its production and implementation is of low cost. And embedding the pros of other related technology can increase the productivity which can be used to recharge a battery pack in less duration. In the present era of electric vehicle implementation need for stored electrical energy reserve is increasing but its supply chain is not developed efficiently. To replace the petroleum-based vehicles completely the electric battery supply system should be implemented in large and efficient quantity with low investments. The main issue is of recharging the battery which is economically infeasible with domestic power supply system. In present digitalized world these resources are expected to be a mobile service and automated system. In this paper we mainly propose an effective solution for these problems with the use of image processing and robotics technologies. In the paper the solution is validated implementing a prototype of the proposed system and presenting its performance analysis.
\end{abstract}

Keywords:- Solar Tracker-Bot, Solar Vehicle, MobileBattery Systems, Raspberry Pi Robot,Image Processing In Vehicle,Electric Vehicle Supply,Solar Charging.

\section{INTRODUCTION}

Energy generation and Transmission is the significant area in present era of decentralization and mobilization. Shortage of non-renewable fuel in the future is an upcoming global issue. The major fact is the depletion of fossil fuel reserves which serves the primary source for industrial and transportation sector and even the coal which is source of thermal electricity station. As the famous saying that a country's modernization or advancement can be measured by its production of consumption of energy, hence the need of energy is ever increasing and it is to be sustained by alternate sources of production.
There are many alternative renewable energy sources like Tidal, Geothermal, Solar etc. Amongst all these sources many are unreliable like wind-based system and tidal systems are mainly depends on unpredictable wind currents. The hydro-generators need sophisticated structural construction to gain the required kinetic energy. Although geothermal is infinitely abundant the heat extraction process is technically not feasible and is also expensive. By all the consideration solar based energy generation systems are reliable replacements for the non-renewable fossil fuels.

Usage a product in huge quantity obviously reduces its cost as there will be less amount spent on storage, hence in these days the electricity costing has been reduced because of high demand and even the production capacity has been tremendously increasing. All fossil fuel-based appliances are modified to operate based on electric energy e.g. lamps, cookers, heaters etc. The main sector of fossil fuel usage are industrial machines and transport systems the industrial machines are mostly converted into electric based by using high voltage current supplies. Even the transportation vehicles are slowly converting into electric major petrol/diesel vehicles are converting into batterybased electric vehicles. But the major drawback is the inconvenient supply of battery wattage which needs to be regularly charged. The charging process consumes a lot of time hence the battery banks concept is gaining ground instead of existing fuel pumps. Although this solution $s$ feasible it is not sustainable and economical because of the need to maintain the reserve battery for long trips and irregular availably of battery banks. Hence in this paper we discuss a solar based mobile battery scavenging vehicle.

As the solar radiation is available for nearly 20 long hours in a day by using suitable booster system to overcome radiation intensity variations the solar energy can be harnessed in very large quantity. The solar radiation is converted to the electric energy by means of the semiconductor-based devices called the Photovoltaic cells. Energy conversion capacity of these cells are based on the radiation incident on it and also on the chemical composition of the cells. The energy conversion process in the photovoltaic cell is due to conversion of photons into electron-hole pair as shown in the fig 1. The energy produced is proportional to the duration and radiation intensity of the sunlight. Hence the production capability can be incremented by exposing the maximum surface of the tee photovoltaic cell to the sun's direct radiation spectrum. Many solar energy harvesting plants use this 
principle either by setting up the stationary array of solar cells faced towards the direction where the solar radiation is available highly in the day or develop a tracker system along with the rotary system which rotates the solar array in the direction of maximum solar radiation as shown in fig2.

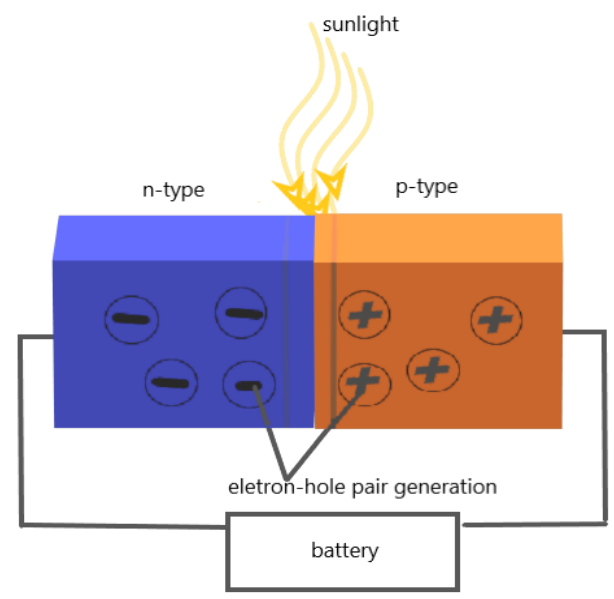

Fig 1:- Photovoltaic cell

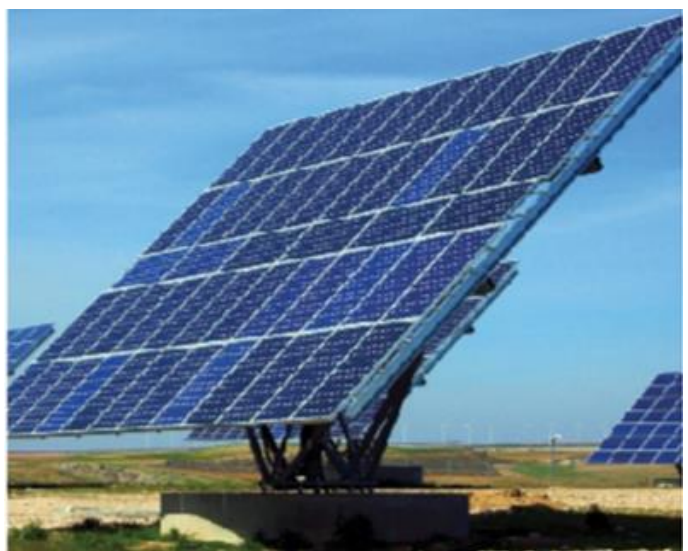

Fig 2:- Tracker-based solar array

Solar cells are traditionally fixed on rooftop or fixed on ground. As a result, solar cells unable to receive maximum light as position of sun changing varying with time. Nearly 2,50,000 metric tons of solar panels are not used efficiently in the world. This leads to the tracker-based system to be implemented and the mobility based scavenging panes are the recent development in energy harnessing field. The scavenger solar array is the combination of tracker-based rotation and the land locomotion systems i.e. the locomotive part moves the array to the open land area where it can get direct exposure to solar radiation and have no problems like shadowing and the tracker system provides the maximum energy generation. The tracer system is available in multiaxial variants like the single axis and dual axis as shown in fig 3. Even the light sensing is done either with active or passive sensors. In the proposed system it is done by means of active camera module using the Image Processing algorithms.

\section{1) Single axis tracker}

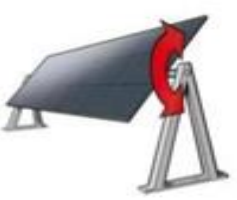

\section{2) Dual axis tracker}

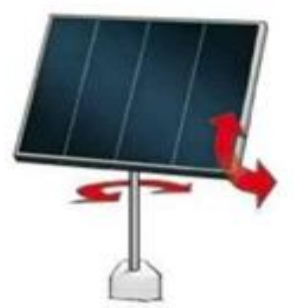

Fig 3:- Types of solar tracker

The paper is organized in the following manner, the challenges of present-day battery powered transport system is discussed in section II. The overview of the proposed solution and its implementation along with requirements are discussed in section III. Obtained results and the uses of the designed prototype is mentioned in the section IV. Section V and section VI consists of the concluding remarks and future scope.

\section{PROBLEM STATEMENT}

The depletion of the fossil fuels made the entire world to move onto the other alternative sources. The main consumption area of this fuel is the transportation system, hence many countries are striving and investing onto electric based vehicle. But the required amount of electricity is a secondary source of energy which has the primary source of coal which is a fossil fuel. Even charcoal can be cheap less efficient primary source but it is environmentally not feasible to produce such amount of charcoal by shedding trees. The fossil fuel less and highquality production of the electricity is the need because with increase in population the present production amount become scares hence the renewable source usage is a need. The availability of the renewable sources is 8abundant but utilization quantity is low. A significant portion of future renewable energy could go to waste without careful planning. A recent report by renewable energy consulting firm Bridge to India (BTI) said that the solar photovoltaic (PV) panels waste volume in India is estimated to grow to 200,000 tonnes by 2030 .

There is a lack of smart technological advancement in order to increase and monitor the production capability of a solar cell. Even the solar power harvesting plants are limited to certain limitations like low level radiation tracking and shadowing effects. With reference to electric vehicle system it is an unreliable source to power up due to speed and mileage limitation of the batteries. Although the battery technology achieved higher improvement in yield duration and power the recharging part is still lagging behind. The charging duration of the battery is less flexible due to its electrolytic nature in lead acid batteries and electron flow requirement of Lithium ion batteries. The efficient way to overcome this limitation is the replacement of battery at refill stations. But the availability of the refill station could be limited due to its maintenance conditions 
and production costs. At an emergency situation the battery cannot be carried as the present petrochemical fuels because of its flammability due to shorting and because of heavier weight which is an overhead. Incase of such a service it is manual task which again doubles the cost of the service. All these limitations can be overcome by the proposed INTELLIGENT SOLAR TRACKER VEHICLE FOR SUPPLY CHANNEL. where it operates in area of maximum sun light availability using tracker and scavenging technologies aided by image processing algorithms. An emergency service is also possible by means of GSM commanding and traffic sensibility based on image processing.

\section{PROPOSED SYSTEM}

\section{A. Basic Idea}

The basic idea behind this prototype is the integration of embedded robotics, Image processing and energy generation technologies. All these technologies are tied upon to a larger space of connecting technologies of Internet of things (IOT) and Machine learning (ML). The learning data for the machine learning are the image datasets of traffic signboards and the basic sound recognition algorithms are used to get the signaling of horn and indicators.

The solar panel is setup with the tracker system consisting of rotary actuator which orients the panel in the angle of solar radiation where refraction is achieved in maximum quantity (angle of production (AP)). The scavenging route is mapped on to the land actuators by means of image processed data of surrounding area captured by the webcam. The data of the vehicle position is tracked on and transmitted to the owner/operator by the use of mobile network in the short message service (SMS) format.

Here the vehicle tracking and energy monitoring are done by means of IOT and solar tracking is done by means of Image Processing (IP) algorithms. The vehicle movement is automated using robotics and the traffic signal following system is done using machine learning.

\section{B. System Overview}

The system aims on the use of the maximum solar energy for powering of the battery with the help of an automatic solar tracking system with an intelligent system which allow to imbibe the traffic rules into the system process. The intelligent sensing system allows the battery status to be regularly updated. The unique feature of the proposed system is that instead of taking the earth as its reference, it takes the sun as a guiding source. The structure of the designed prototype system is mechanically stabilized so that the carrying capability and spacing is maximized to be used as a battery carrier or local transporter system.

The system block diagram is as shown in the fig 4 . The system has a central controlling unit (CCU) which is a Raspberry PI(R-pi) in the system along with secondary controlling unit (SCU) as Arduino UNO opensource controller which have a slave relationship with the central controller. This master-slave combination manages the external communication systems. In this system there are two external communication systems like the ZigBee node and the subscriber identity module (SIM) enabled GSM. Where GSM directly interacts with master and the Zigbee interacts with the slave and used to transmit the minor information and generation status to the operator/owner.

The relay module array controls the speed of the DC motors hence controls the accuracy of the vehicle movement and its precision. Th relay decides the input voltage by the processed information received by the CCU. The infrared (IR) sensor is directly senses the environment into the Master controller. The IR sensor is used to detect the obstacles like rock, wall, other vehicle with low accuracy where it acts as a supplement to reception of data left out by the camera interface. The camera interface is a wired optical sensor which captures the optical data of the surrounding and pushes it into the CCU. CCU performs all the image processing out of the red, green, blue (RGB) format of data captured by the optical sensor.

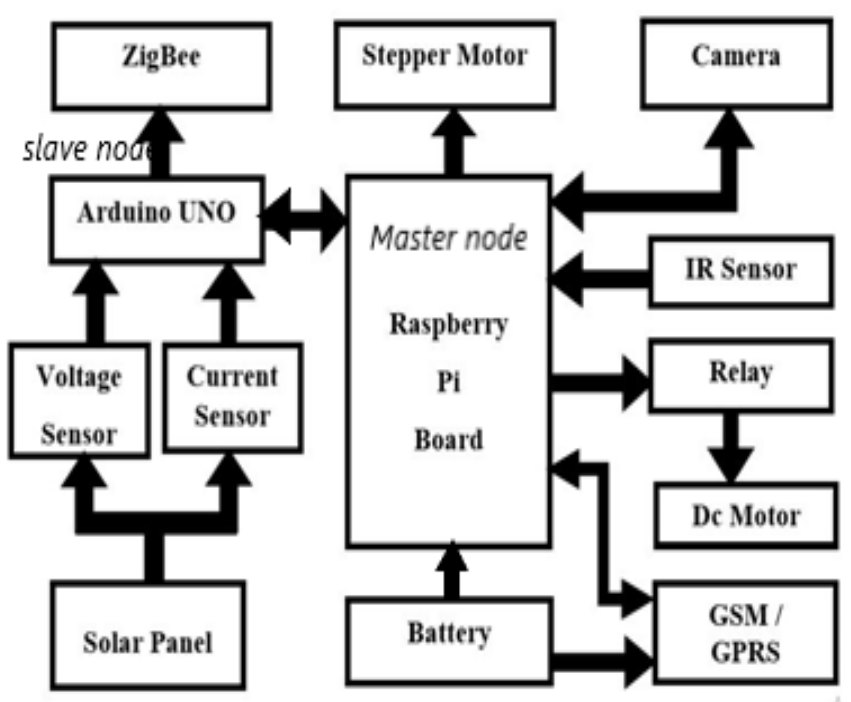

Fig 4: Block Diagram of the Proposed system

The camera setup is coupled with the stepper system which rotates the camera in required angular rotation to a full capture of a circle i.e. $360^{\circ}$ the stepper is given $30^{\circ}$ rotation steps. The stepper system even controls the tilting system of the solar panel. A solar tracker stepper device can orient a payload toward the Sun. Payload is solar panel in this prototype design other payloads can also be used are like parabolic troughs, Fresnel reflectors, lenses or the mirrors of a heliostat. The generated wattage in solar panel is sensed by the current and voltage sensors which generates an analog value that is converted into digital numbers by the SCU and based on this value the master system decides the precision of the tilt in solar panel. The information of voltage and current is transmitted through ZigBee. 
GSM provides a queued duplex communication whereas the ZigBee supports half-duplex mode of communication. The data transfer direction and modes are shown with arrows in fig 4. Even the camera module supports the half duplex mode. The only full duplex wired communication happens between master and slave controller systems.

\section{Requirement and Interfacing}

The Hardware requirement of the prototype are as shown in fig 5. The controlling units used are the Raspberry PI 4 model $B$ which is a 2 GB RAM variant. And the Arduino UNO board. The Raspberry Pi is a series of small single-board computer. The Raspberry Pi 4 uses a Broadcom BCM2711 system on chip (SoC) with a $1.5 \mathrm{GHz}$ 64-bit quad-core ARM Cortex-A72 processor, with $1 \mathrm{MiLB}$ shared L2 cache, the interrupt controller on this SoC is compatible with the ARM generic interrupt controller (GIC) architecture 2.0. Arduino Uno is a microcontroller board based on the ATmega328P. It has 14 digital input/output pins (of which 6 can be used as PWM outputs), 6 analog inputs, a $16 \mathrm{MHz}$ ceramic resonator, a USB connection, a power jack, an ICSP header for serial programming and a reset button.

The wireless communication to external system is done with two communication devices one is ZigBee Transceiver that works on ISM band (2. 4 Ghz) reserved internationally that do not require any license. It supports multiple frequencies within same band rate that helps in avoiding data collision with no requirement of complex wireless connection software for connecting to serial devices. The Global System for Mobile Communications (GSM) is a standard developed by the European Telecommunications Standards Institute (ETSI) to describe the protocols for second-generation (2G) digital cellular networks. SIM800 is a complete Quad-band GSM/GPRS solution in a SMT type which can be embedded in the customer applications. Which support Quad-band $850 / 900 / 1800 / 1900 \mathrm{MHz}$, it can transmit Voice, SMS and data information with low power consumption.

As the main principle used here is the IOT it consists of multiple sensors and actuators. The sensors used are IR sensor, Current sensor and Voltage sensor. The IR sensor is a reflection-based sensor which emits infrared radiation and detects obstacle or distance of the obstacle by the received reflected infrared signal. It supports both analog and digital writing. A current sensor is a device that detects electric current in a wire and generates a signal proportional to that current. It measures both alternating and direct currents. Current sensor uses the famous ACS712 IC to measure current using the Hall Effect principle. Voltage Detection Sensor Module is a simple and very useful module that uses a potential divider to reduce any input voltage by a factor of 5. This allows us to use the Analog input pin of a microcontroller to monitor voltages higher than it capable of sensing. The actuators used are DC motors of specification of $12 \mathrm{~V}$ Johnson family center shaft with 100 $\mathrm{rpm}$ of speed. DC motor is controlled by means of a commuter/regulator array of relay switch. Another actuator is stepper which produce precise angular rotation in stepwise motion.

A webcam is an optical sensor that feeds or streams an image or video in real time to a controller. The maximum resolution of a webcam is also lower than most handled video cameras, as higher resolutions would be reduced during transmission. As the resolution of the camera increases the quality of data processing.

Solar modules are currently produced from crystalline silicon (c-Si) solar cells made of multi crystalline and mono crystalline silicon. The solar panel is selected based on the production requirement. In this prototype we used a rigid frame system. Even flexible frame, foldable cell array is available.

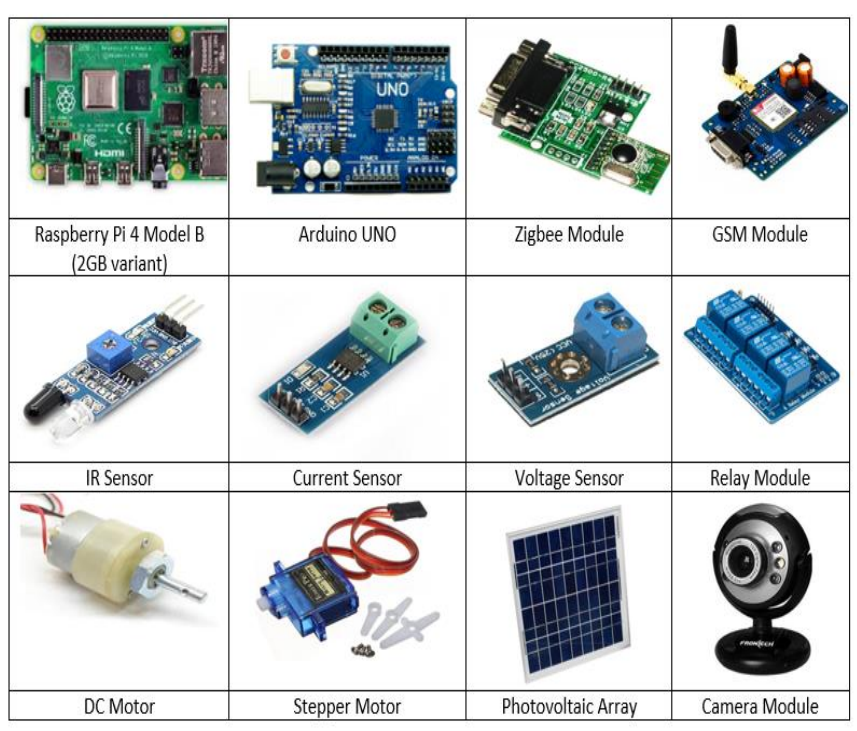

Fig 5:- Hardware used in this project

The software used in this protype programming are Python IDLE from www.python.org, for coding the CCU and image processing by using OpenCV library. Arduino IDE for coding Arduino UNO provided by www.arduino.org. The APK from play store named IP PHONE CAMERA is used to get the live video and audio of the vehicle surrounding as a pilot security.

Interfacing plays an important role in correct usage of any system. As mentioned in the previous section the main vehicular structure is based on the robotics components. In this prototype the infrared, current and voltage sensors are used. The IR sensor is directly connected to Raspberry Pi module provid0ing the digital input whereas the rest two i.e. which provide analog output based on panel input are connected to Arduino controller which in turn connects to the ZigBee node for remote data transmission. The robot locomotors i.e. DC motors are interfaced to raspberry pi by means of the relay arrays as shown in fig 6 . The solar panel generated wattage is used to charge the battery. Stepper and camera combo are connected to raspberry pi where camera is interfaced using the USB port and the stepper is connected to its digital GPIO pin. The GSM module is 
connected to raspberry pi by means of VDI to USB cable. All the connections are established out of insulated jumper cords. The powering system line is defined as below:

$>$ Solar panel $\rightarrow$ Battery $\rightarrow$ Raspberry $\mathrm{Pi} \rightarrow$ Other peripherals (relay, IR sensor, Arduino, GSM, camera module).

$>$ Arduino $\rightarrow$ peripherals (current sensor, voltage sensor, Zigbee node)

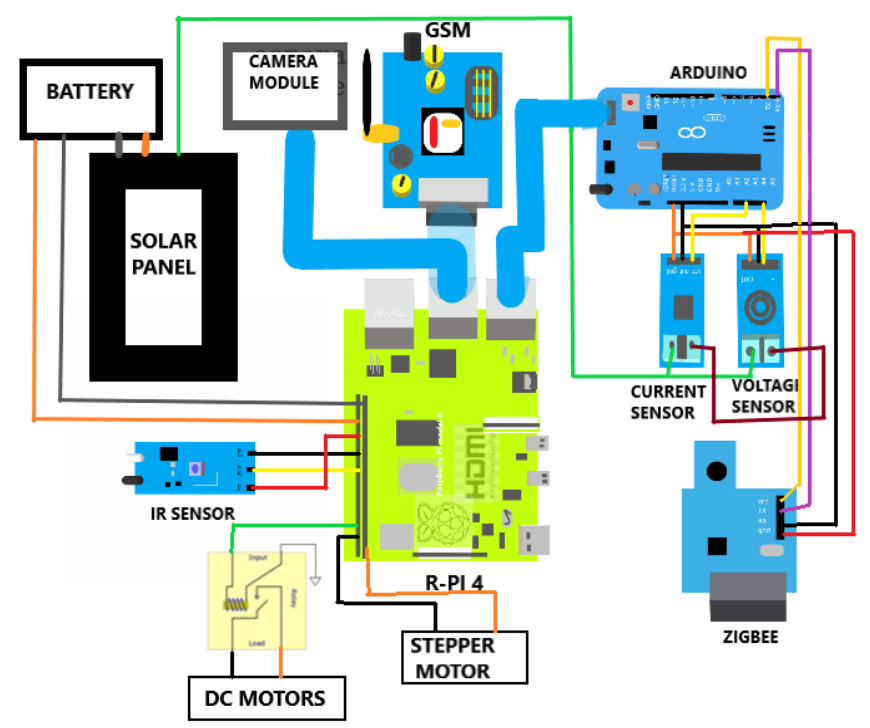

Fig 6:- Circuit interfacing diagram

\section{Algorithm and Programming}

Different algorithms are used in the working of different components. In this section we discuss the algorithms used by hardware and the main image processing algorithm.

Firstly, the communication interface of GSM uses GPS (Global Positioning System) as the reference that furnishes location and time information in all climate conditions to the user. At least 4 GPS satellites are 'visible' at any time. The operation of the GPS is based on the 'trilateration' principle. The position is determined from the distance measurements to satellites. Three satellites out of four are used to trace the location place. A fourth satellite is used to confirm the target location of each of those space vehicles. The global positioning system consists of satellite, control station and monitor station and receiver. The GPS receiver takes the information from the satellite and uses the method of triangulation to determine a user's exact position.

Secondly, another communication interface Zigbee works on the principle of IEEE 802.15.4 standards. It creates a personal area network with small, low powered nodes of its same kind and transmits the data wirelessly to the destination node by means of smallest hop paths. Even Ad-Hoc networks can also be generated in case of multiple command stations to send and receive commands by all verified battery stations.
Finally, the main algorithm of image processing works on the coding of python language using a sophisticated OpenCV library. Images are captured using the camera module and these images are stored as pixels values, where each pixel value represents a color intensity value. In this system there are two algorithms are used on to the captured image one is for determining the brightness and the other to read traffic signals. The brightness of images is obtained by using HSV comparison technique in which image is converted into grey scale image using dithering method and using histogram of gray scale images brightness is calculated. Gray level is a level in which all three RGB gradients are equal in composition. The gray scale conversion is done using Weighted method or luminosity method. Since red color has more wavelength of all the three colors, and green is the color that has not only less wavelength then red color but also green is the color that gives more soothing effect to the eyes. It means that we have to decrease the contribution of red color, and increase the contribution of the green color, and put blue color contribution in between these two. It can be done by applying the following equation to every pixel. Conversion is as shown in fig 7 and the equation is:

$$
\text { Grayscale image }=((0: 3 R)+(0: 59 G)+(0: 11 B)):
$$

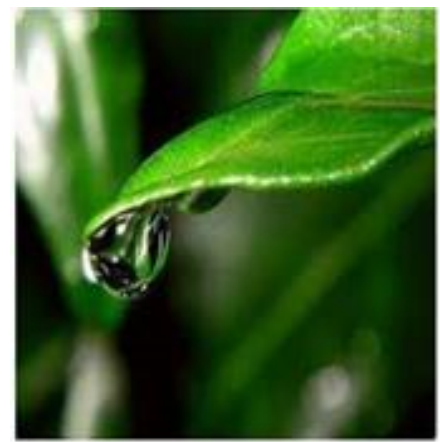

a) Original image

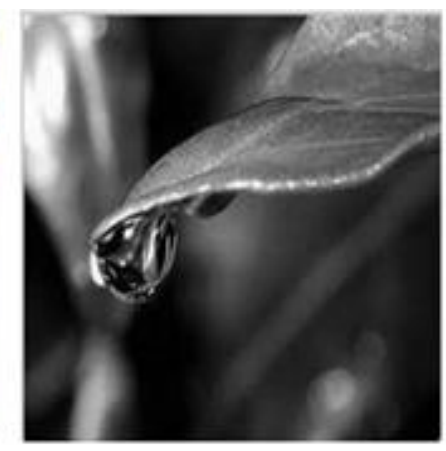

b) Converted image
Fig 7:- RGB to Gray conversion

Dithering is majorly help to improve thresholding. During thresholding, the sharp edges appear where gradients are smooth in an image. In thresholding, we simply choose a constant value. All the pixels above that value are considered as 1 and all the value below it is considered as 0 . Since there is not much change in the image, as the values are already 0 and 1 or black and white in this image. even its contrast is very low. Hence for the first-time dithering is done more information is obtained and on second dithering the contrast improves as shown in fig 8 . 

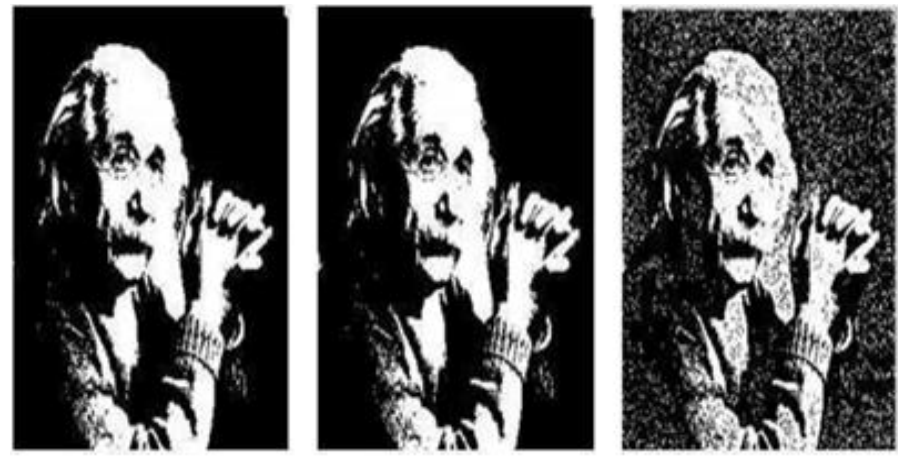

(a) Image with less information (b) Dithered image1

(c) Dithered image 2

Fig 8:- Dithering Method.

This image is then thresholded to get a required information in area of interest this image is then converted into histograms. An image histogram is a type of histogram that acts as a graphical representation of total distribution in a digital image. More precisely, this histogram is a graph showing number of pixels in an image at each different intensity values found in that specific image. These histograms of different images are compared with one another and the brightness spot is determined by difference method and the vehicle receives the signal to move towards that spot.

The traffic signal information s obtained by the same image by the combination of object detection and support vector machine algorithm combination. In the image certain objects of certain shapes are highlighted and extracted these images are then classified using SVM algorithm to traffic set and neglect set the image in traffic set is again given to object detection algorithm 2 which finds the character words and symbols and map it into certain commands to be taken in traffic. The horn and indicator sounds are detected using diaphragm system and decoded into respective command.

The proper working of individual components is tested before programming. Once the components are assembled the Arduino is programmed in $\mathrm{C}++$ language using IDE software for analog to digital conversion of sensor data and for interaction with CCU. The Raspberry Pi is coded using Python language which include motor controlling, GPIO pin usage description, brightness calculation code and traffic signal detection code with their respective interconnections.

\section{OUTCOME AND ANALYSIS}

\section{A. Result Obtained}

The vehicle is implemented and tested on a synthetic small test environment with designed paths and traffic signal/symbols and the light sources of various illumination are setup as a reference of sun light. A registered mobile network card (SIM) has been used for GSM tracking.

The structure of the vehicle is as shown in the fig 9. The prototype design is mechanically stable and it provides maximum spacing capacity and it is structurally balanced so that the energy generation panel can easily moved in multiple direction and even it must suffer less jerking effect due to unequal road surface. The controller and its peripherals are placed such that they do not suffer any physical stress and peer care is taken so that the connection remain intact. It is observed that the motor commuter relay response time is so minimum that the path tracing action is nearly real time in function. The working of tracker stepper and camera stepper is efficient with precise rotation hence adding to production of energy and production of image data.
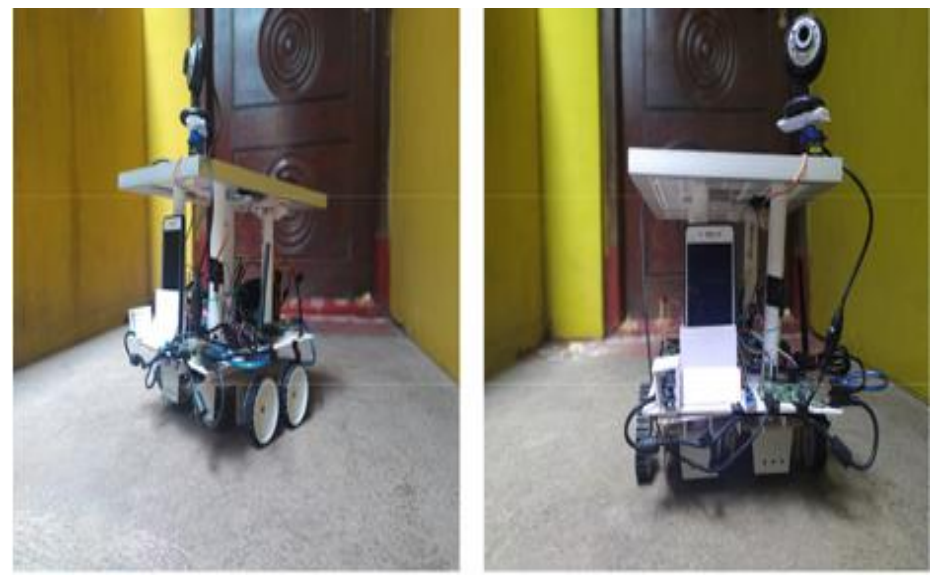

Fig 9:- Side view and Front view of the vehicle structure

The images captured by the webcam are processed using HSV method and the decision taken is verified by means of the vehicle movement direction. The brightest image among multiple captured image is verified and mapping to respective command is done successfully. The capturing and average brightness of each image is indicated on the R-pi display as shown in fig 10. In the below figure the location details of each snap is also displayed.

\section{Brightness of 1st Snap 0.5787432861328123 \\ Brightness of 2nd Snap 0.6090105692545578 \\ Brightness of 3rd Snap 0.5333417256673182 \\ Brightness of 4 th Snap 0.5151187642415366 \\ Brightness of 5 th Snap 0.4192742411295569 \\ Brightness of 6 th Snap 0.41810201009114534 \\ 12}

$K \backslash r \backslash n ' b^{\prime} A T+C G P S I N F=$

Robot is at $13.947015,75.5742780^{\circ}$

message sent

c2

Fig 10:- Brightness detection process

Once the vehicle moves towards the brightest area it then starts orienting the solar panel such that the production maximize. This is internally done by the CCU unit based on slave device feedback. In this system the analog to digital conversion (A to D) is efficiently done by the slave device Arduino for very slight data change. The real time current and voltage produced in solar panel is displayed on the Arduino console as shown in fig 11. The data displayed are the raw analog data from the voltage and current sensors. 
This data is wirelessly transmitted between the two Zigbee node one in the vehicle another in the operator's system.

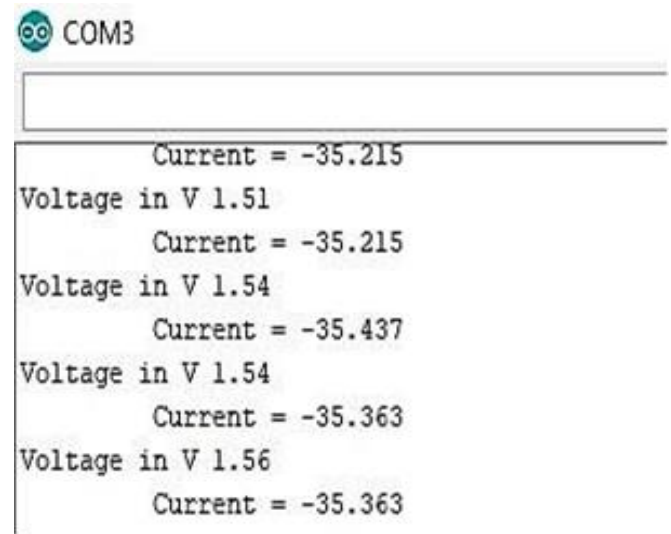

Fig 11:- Real time current and voltage value display

The real time location of the vehicle is updated regularly in fixed interval of time through SMS using the SIM enabled GSM system. The location is specified by mean of $\mathrm{X}$-axis and $\mathrm{Y}$-axis position converted into a location Id. In this system only GSM is the earth centric device which aligns into longitude and latitude of the earth. Even the destination location can be sent in the format for service delivery with high priority operation. This function yielded the accuracy of 80 percent. The updating of vehicle location is as shown in fig 12 .

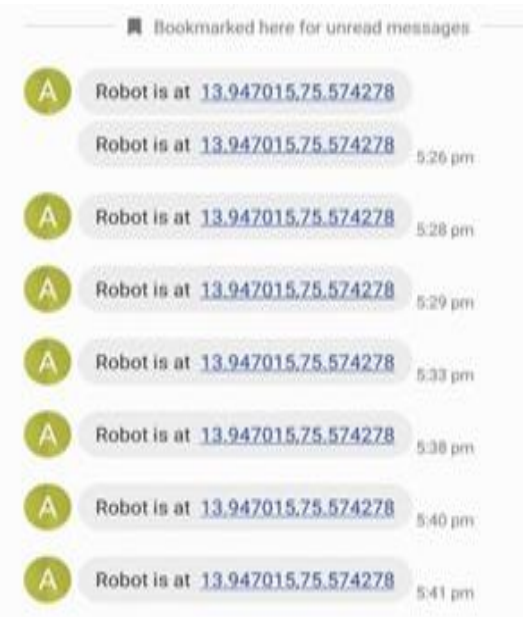

Fig 12:- GSM SMS location updated

The traffic signal processing is achieved to be 90 percent efficient system. The 10 percent error occurred due to unclear images and untrained data. This system can be improved with implementing high resolution camera and higher precision dataset usage. The pilot security implementation of IP based real time video and audio data acquisition is achieved with high efficiency providing a hope of shift to secure internet channel system of distributed processing. By which the processing delay on $\mathrm{CCU}$ is reduced to maximum extent. The pilot trail result is shown in fig 13.

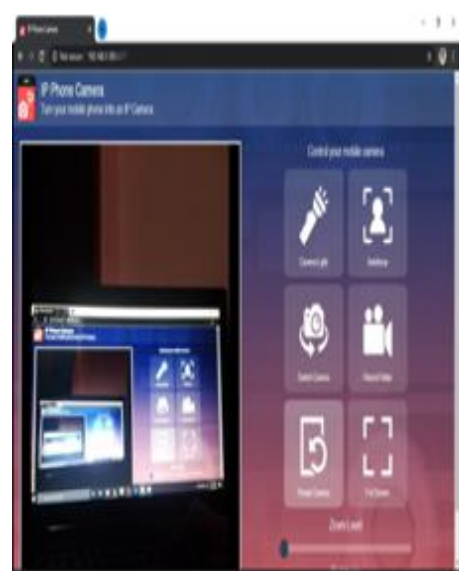

ISSN No:-2456-2165

Fig 13:- Real time audio video transmission using

\section{IP PHONE CAMERA APK}

\section{B. Advantages}

The proposed locomotive system is advantageous in many ways. It can generate more electricity than their stationary counterparts due to an increased direct exposure to solar rays. There are many different designs of solar tracker, such as single-axis and dual-axis trackers, which can help you find the perfect fit for your unique job site based on environmental and maintenance factors. Other advantages are as below:

$>$ Environment friendly.

$>$ Zero Energy overhead.

$>$ Economical.

$>$ Efficient manpower utilization (Semi-automated process).

$>$ Increases Customer satisfaction.

\section{Limitations}

As every product have its pros and cons there are some areas of limitation in the proposed prototype system which can be improved on time. some of the disadvantages of the system are as follows:

$>$ Solar trackers are slightly more expensive than their stationary counterparts.

$>$ Implementing the specific traffic sense within the vehicle is a programming complexity.

$>$ Multi functionality processing of image data may lead to image degradation.

$>$ It is highly based on mobile network; hence network drop is a major issue.

$>$ The battery maintenance in all-weather condition is to be done properly or else leads to battery failure.

$>$ The security issues associated with payments and battery replacements are the ethical drawback.

\section{Applications}

As mentioned earlier it can be used as the power generator system for the local battery stations and can also serve as a mobile battery station for the electric vehicle with GSM based delivery service. Some of the other applications of proposed systems are listed below:

$>$ Power generation unit in remote areas.

$>$ Can be used for commodity delivery system like amazon, flipcart as a local delivery. 
- Can be used in malls as automated follower cart.

$>$ Can be used as a lightweight local domestic transport system.

Used as water sprinkler in low water irrigation systems.

Solar tracking bot is applicable in Military Applications.

\section{CONCLUSION}

The concept avoids wastage of solar panel efficiency and increase the electricity production in smart manner, Solar panels are very expensive so proper usage of them will be optimistic. The idea of producing solar energy using tracker technology is an existing technology, but the proposed system aims at a general solution to transportation and energy sustainability issues. Its main business objective and lending support system to the emerging electric vehicle industry in transportation platform. The effective utilization of existing technologies and its integration to create the unique yet effective solution for energy sector is the main motto of this system which is partially achieved and the further development is expected in the near future. At the end of the paper we would like to conclude it with the achieved success and aim of further improvement on the basic ideology. The system fulfilled all the objectives it supposed to be served.

\section{DISCUSSION}

The proposed system provides a strong framework on which vivid future works can be carried out. The framework of present system is a combined technology and it has the scope of inclusions of many other technologies into it. The framework can be designed in various specifically design to be operated in different remote areas like mountains, oceans etc...

The work specific design can be developed for small transportations e.g. solar auto rickshaws and industrial robots e.g. solar aggregator (pick and pile). Even the technology can be directly implemented onto electric cars making it energy self-sustainable. The vehicle can be modified efficiently into an automatic agricultural vehicle because of less work duration and requirement of impulsive forces as in tractor tillers etc. It can be developed as the all=weather compatible vehicle and can be easily used in war zone as self-sustainable supply and goods carrying mule robots.

\section{REFERENCES}

[1]. K.S. Shilpashree, Lokesha, Hadimani Shivkumar, Implementation Image Processing on Raspberry $\mathrm{Pi}$, International Journal of Advanced Research in Computer and Communication Engineering Vol. 4, Issue 5, May 2015.

[2]. Hui Liu, Chenhui Yang, Xiao Shu, Qicong Wang, A New Method of shadow detection based on edge information and HSV color information, 2009 2nd International Conference on Power Electronics and Intelligent Transportation System.
[3]. Ching-Hung Su, Huang-Sen Chiu and Tsai-Ming Hsieh, An encient image retrieval based on HSV color space,2011International Journal of Current Engineering and Technology.

[4]. Kunal Maurya, Mandeep Singh and Neelu Jain, Real Time Vehicle Tracking System using GSM and GPS Technology-An Antitheft Tracking System, January 21, 2020 Computer Vision Laboratories.

[5]. Cheng-Dar Lee, Hong-Cheng Huang and Hong-Yih Yeh," The Development of Sun-Tracking System Using Image Processing", Sensors 2013, 13, 54485459; doi:10.3390/s130505448.

[6]. Tutorial for TSBB15, A review of robust image enhancement algorithms and their applications, 2016 the 4th IEEE International Conference on Smart Energy Grid Engineering.

[7]. Rajwant Kaur, Sukhpreet Kaur," Object Extraction and Boundary Tracing Algorithms for Digital Image Processing: Comparative Analysis: A Review", International Journal of Advanced Research in Computer Science and Software Engineering, Volume 3, Issue 5, May 2013

[8]. Morteza Taki, Yahya Ajabshirchi, Hossein Behfar, Mohsen Taki," Experimental Investigation and Construction of PV Solar Tracker Control System Using Image Processing”, Modern Applied Science, Canadian Center of Science and Education, Vol. 5, No. 6; December 2011.

[9]. C.Saravanan, Dr.M.A. Panneerselvam, I. William Christopher, "A Novel Low-Cost Automatic Solar Tracking System", International Journal of Computer Applications (0975 - 8887), Volume 31- No.9, October 2011

[10]. Gang Jun Tu, Henrik Karstoft, Line Juul Pedersen and Erik Jorgensen, "Illumination and Reflectance Estimation with its Application in Foreground Detection"

[11]. Mehmet Kayakuş, Ismail serkan Üncü Article in Przeglad Elektrotechniczny, January 2010 "Luminance and illuminance analysis of roads using image processing technique"

[12]. Adam Kaplan, Paul Uhing, Nathaniel Kingry \& Randai "Integrated Path Planning and Power management for solar-powered Unmanned Ground Vehicles",2015 IEEE International Conference on Robotics and Automation (ICRA).

[13]. P. Jothimurugan, J. Muthu Saravanan, R. Sushanth, V. Suresh, H. Siva Subramaniam, S. Vasantharaj., S. Yogeswaran, "Solar E-Bot for Agriculture", Sri Eshwar College of Engineering, Coimbatore. 